\title{
Shear wave-based sound touch elastography in liver fibrosis assessment for patients with autoimmune liver diseases
}

\author{
Lulu Yang ${ }^{1}$, Wenwu Ling ${ }^{1}$, Du He${ }^{2}$, Changli $\mathrm{Lu}^{2}$, Lin $\mathrm{Ma}^{1}$, Lin Tang ${ }^{1}$, Yan Luo ${ }^{1}$, Shigao Chen ${ }^{3}$ \\ ${ }^{1}$ Department of Ultrasound, West China Hospital of Sichuan University, Chengdu, China; ${ }^{2}$ Department of Pathology, West China Hospital of \\ Sichuan University, Chengdu, China; ${ }^{3}$ Department of Radiology, Mayo Clinic, Rochester, MN, USA
}

Correspondence to: Yan Luo. Professor, Department of Ultrasound, West China Hospital of Sichuan University, 37 Guoxue Lane, Chengdu 610041, China. Email: luoyanddoc@163.com.

\begin{abstract}
Background: Shear wave-based ultrasonic elastography (USE) has been widely used for the assessment of liver fibrosis in patients with chronic liver diseases (CLD). However, diagnostic criteria and accuracy vary between different etiologies and specific elastography techniques. We aimed to evaluate the tissue stiffness measured by shear wave-based sound touch elastography (STE) in staging liver fibrosis in patients with autoimmune liver diseases (AILD).

Methods: One hundred and two AILD patients who had undergone STE liver stiffness measurements (LSMs) by using a Resona 7 ultrasound system were retrospectively studied. With the Scheuer liver fibrosis staging system as the reference, we investigated the diagnostic performance and cutoff values of STE measured liver stiffness in staging liver fibrosis through receiver operating characteristic (ROC) curve analysis. Moreover, comparisons of areas under the curve (AUCs) were made between LSMs and calculated biomarker scores, including the aspartate aminotransferase (AST)-to-platelet ratio index (APRI), and fibrosis-4 (FIB-4) index.
\end{abstract}

Results: Median LSMs increased with the advancing fibrosis stages with values of $6.89 \mathrm{kPa}(1.51 \mathrm{~m} / \mathrm{s})$, $8.00 \mathrm{kPa}(1.63 \mathrm{~m} / \mathrm{s}), 9.60 \mathrm{kPa}(1.79 \mathrm{~m} / \mathrm{s}), 11.37 \mathrm{kPa}(1.95 \mathrm{~m} / \mathrm{s})$ and $14.50 \mathrm{kPa}(2.20 \mathrm{~m} / \mathrm{s})$, from stage 0 to stage 4 respectively. The cutoff values of STE for identifying significant fibrosis ( $\geq$ stage 2$)$, severe fibrosis $(\geq$ stage 3 ) and cirrhosis (stage 4) were $9.07 \mathrm{kPa}(1.74 \mathrm{~m} / \mathrm{s}), 9.97 \mathrm{kPa}(1.82 \mathrm{~m} / \mathrm{s})$ and $10.48 \mathrm{kPa}(1.87 \mathrm{~m} / \mathrm{s})$, respectively, with corresponding sensitivity of $79.1 \%, 93.3 \%$, and $100 \%$; specificity of $80.0 \%, 70.8 \%$ and $71.8 \%$. The AUCs of LSMs in identifying fibrosis $\geq$ stage $2, \geq$ stage 3 and stage $4(0.82,0.87$, and 0.91 , respectively) were significantly higher than that of APRI $(0.67,0.64$, and 0.72 , respectively) and FIB-4 $(0.70,0.68$, and 0.75 , respectively) (all $\mathrm{P}<0.05)$.

Conclusions: LSM obtained by STE exhibited its good capability to evaluate liver fibrosis stages in patients with AILD. As a noninvasive modality for liver fibrosis staging, STE is superior to APRI and FIB-4 biomarker scores.

Keywords: Autoimmune liver disease (AILD); liver fibrosis; liver stiffness; shear wave elastography (SWE); sound touch elastography (STE)

Submitted Apr 01, 2020. Accepted for publication Oct 30, 2020.

doi: 10.21037/qims-20-521

View this article at: http://dx.doi.org/10.21037/qims-20-521

\section{Introduction}

Autoimmune liver diseases (AILD) include a number of diseases characterized by immunity mediated hepatocytes or bile ducts injury, which is always accompanied by abnormally elevated autoantibodies and the possibility of progressing to cirrhosis. Although AILD are relatively uncommon compared with viral hepatitis in the AsiaPacific region, the prevalence is increasing worldwide 
according to recent reports $(1,2)$. Late treatment of these diseases may result in cirrhosis and, ultimately, liver failure. Early identification and accurate staging of liver fibrosis are of great value in medical management, as well as, for evaluating therapeutic responses $(3,4)$.

Currently, liver biopsy is the standard for assessing liver fibrosis. However, it is a costly and invasive procedure, with the risk of complications including bleeding and hemobilia. Additionally, it is susceptible to sampling errors and the inter- or intraobserver variability, which leads to misjudgement of liver fibrosis stages (5-7). Therefore, it is ill-suited to be frequently used in patient screening and follow-up in the clinic. The fibrosis-related serum biomarkers indexes such as aspartate aminotransferase (AST)-to-platelet ratio index (APRI) and fibrosis index based on the four-factor fibrosis-4 (FIB-4) index have been used as noninvasive approaches to identify significant or severe fibrosis in chronic hepatitis $\mathrm{C}$ (CHC) patients $(8,9)$. However, their clinical utilities in patients with other etiologies of chronic liver diseases (CLD) remain controversial (10-13).

Liver stiffness measurement (LSM) using imaging modalities is one of the effective and promising noninvasive approaches for assessing liver fibrosis. Currently, shear wavebased ultrasonic elastography (USE) techniques, such as transient elastography (TE), two-dimensional shear wave elastography (2D-SWE), and point shear wave elastography (pSWE) (13-18), have been widely used in the clinic and the LSMs have demonstrated positive correlations with histologic hepatic fibrosis stages in patients with CLD. Nevertheless, the diagnostic criteria and accuracy varied among different etiologies and specific elastography platforms implemented by different manufacturers (13-21). Sound touch elastography (STE) is a relatively novel shear wave ultrasound elastography technique using ultra-wide beam tracking imaging technology, which is compatible with the diagnostic ultrasound device. It provides real-time processing of signals within areas up to $40 \mathrm{~mm}$ wide on one shot and effectively detects the shear wave information as high as $10 \mathrm{kHz}$ per frame. Eventually, it displays high-quality two-dimensional color-coded tissue stiffness imaging and provides quantitative measurements at the same time. To date, studies on assessing the diagnostic performance of STE in liver fibrosis staging have been very limited $(22,23)$. Moreover, none of the studies have focused on the performance of STE in fibrosis staging specifically on patients with AILD.

This study aimed to explore the diagnostic criteria of LSMs obtained by STE and assess its diagnostic performance in staging liver fibrosis in patients who were pathologically diagnosed with AILD including autoimmune hepatitis (AIH), primary biliary cholangitis (PBC), and AIH-PBC overlap syndrome.

\section{Methods}

\section{Patients}

This retrospective study was approved by the Ethics Committee of West China Hospital with written informed consent from all the patients. From February 2019 to January 2020 in West China Hospital, patients who were suspected of suffering AILD and eventually underwent liver biopsy were enrolled in this study. All these patients had performed LSMs before liver biopsy and none were on immunosuppressive therapy when recruited. One hundred and twenty-one patients who were histologically diagnosed as $\mathrm{AIH}, \mathrm{PBC}$, or AIH-PBC overlap syndrome were included. The exclusion criteria were as follows: patients under 18 years old; patients infected with hepatitis virus or accompanied by other liver diseases, such as hereditary metabolic liver disease, drug-induced liver disease; patients whose serum biomarkers were not completely obtained within 4 weeks before biopsy.

\section{LSMs obtained by STE}

LSMs were performed by one of two radiologists (LY and WL) using a Resona 7 ultrasound system (Mindray Medical Solutions, Shenzhen, China) equipped with an SC6$1 \mathrm{U}$ convex array probe $(1-6 \mathrm{MHz})$ and the STE feature. Both radiologists have more than 8 years of experience in abdominal ultrasound scans and 5 years of experience in ultrasound elastography examination. All STE examinations were performed within 7 days before the biopsy. Patients were instructed to fast for 8 hours and rest for at least 10 minutes before the examination. According to the European Federation of Societies for Ultrasound in Medicine and Biology (EFSUMB) recommendation (24), patients were required to lie in the supine position with the right arm raised above the head. Measurements were obtained in the right liver lobe through the appropriate intercostal space during a quiet breath-hold of 4-5 seconds, aimed at achieving stable homogenous color filling of the areas in the acquisition box.

An elasticity acquisition box about 3 to $4 \mathrm{~cm}$ was positioned at the desired area a minimum of $1 \mathrm{~cm}$ and a 


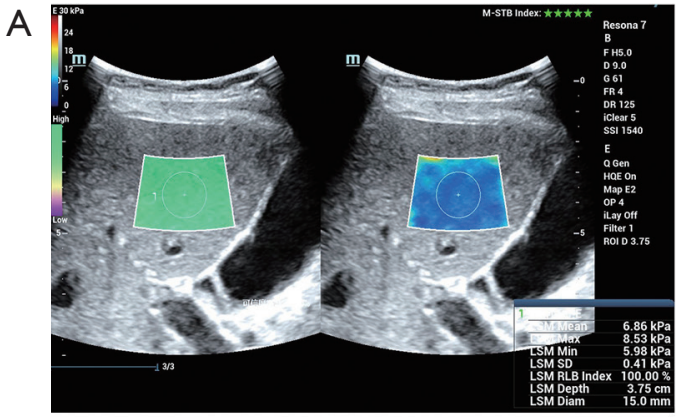

so

B

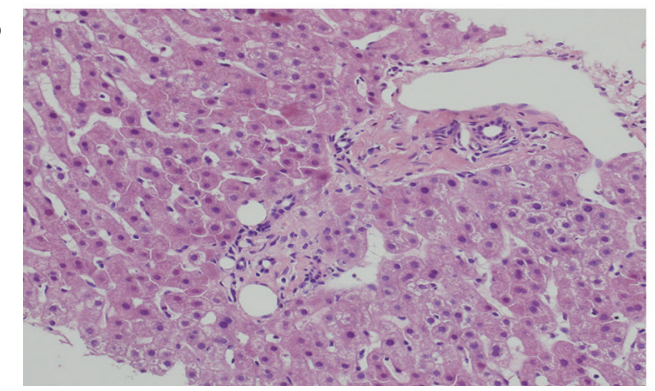

so

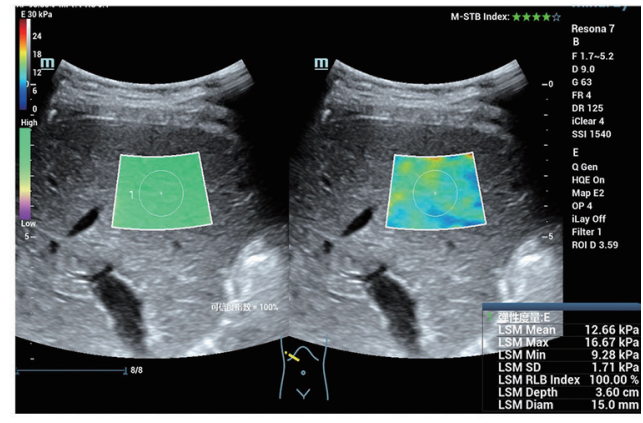

S4

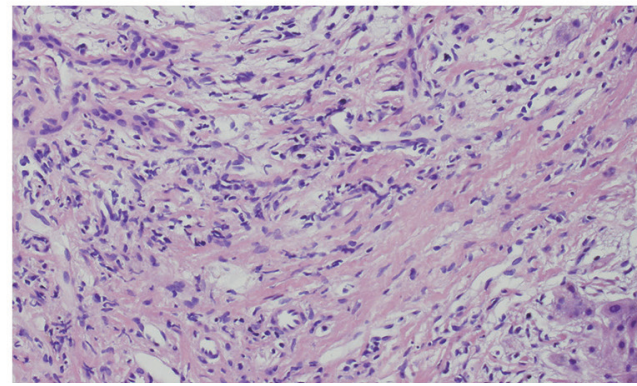

S4

Figure 1 STE examination and pathological Scheuer classification in livers in patients with fibrosis stage (S) 0 and stage 4. (A) STE maps of the right anterior hepatic lobes from a 47-year-old man with liver fibrosis stage of 0 and a 48 -year-old woman with liver fibrosis stage of 4 , with M-STB index and uniform green RLB map presented; (B) pathological hematoxylin-eosin staining images of livers with fibrosis stages of 0 and 4 (original magnification, $\times 20$ ). STE, sound touch elastography; M-STB, motion stability; RLB, reliability.

maximum of $6 \mathrm{~cm}$ beneath the liver capsule right in the middle line of the elastogram, while free of large vessels, gallbladder, and artifacts $(24,25)$. The operator placed a circular analysis box with a diameter of $15 \mathrm{~mm}$ in the relatively homogeneous areas of the acquisition box to measure stiffness. The stiffness values are quantitatively expressed as Young's modulus in kilopascals $(\mathrm{kPa})$. All values were further converted to shear wave speed (SWS) in $\mathrm{m} / \mathrm{s}$. [Young's modulus $(\mathrm{kPa})=3 \times \mathrm{SWS}^{2}$, assuming an isotropic tissue density of $1 \mathrm{~g} / \mathrm{mL}$ ] $(26,27)$. The Resona 7 system offers reliability (RLB) indicators of motion stability (M-STB) index and RLB map. LSMs were considered valid when both the M-STB index displayed at least four stars and the RLB map was uniform green, with an index $>95 \%$ (Figure $1 A$ ). For each patient, three consecutive acquisitions were performed. Median value of the three elasticity measurements was used for further statistical analysis. Technical failure was defined when no valid measurements were obtained from any acquisition. Among the valid measurements, unreliable results were defined as an interquartile range/median (IQR/M) greater than $30 \%(19,24)$.

\section{Liver biopsy and histology evaluation}

Ultrasonography-guided liver biopsy was conducted no longer than 1 week after the STE examination. Samples were obtained from the right liver lobe using 18-gauge core biopsy needles. All included liver specimens should be at least $1.5 \mathrm{~cm}$ in length with at least eight complete portal tracts. For each patient, 2-3 biopsy specimens were collected. All the histological specimens were fixed in $10 \%$ formalin and embedded in paraffin. The biopsy stained slices were further evaluated by two pathologists (CL and DH) blinded to the LSMs results, both with work experience above 10 years in liver pathological diagnosis. The Scheuer grading system was used to assess fibrosis stages and inflammatory activities of the liver (28). Liver fibrosis was scored from stages 0 to 4 , of which stage 0 and 4 were presented in Figure 1B: S0, no fibrosis; S1, enlarged portal fibrosis without septa; S2, periportal or portal-to-portal fibrosis with few septa; S3, bridging 
fibrosis accompanied by distorted architecture; S4, probable or definite cirrhosis with pseudolobule formation. The inflammatory activity was also classified as grade $0,1,2$, 3 , or 4 , presented as degrees of none, minimum, mild, moderate, or severe.

\section{Laboratory test}

The recorded serum biomarkers included: liver biochemistry [i.e., AST; alanine aminotransferase (ALT); gamma-glutamyltransferase (GGT); alkaline phosphatase (ALP); total bilirubin (TBIL), and albumin (ALB)], serum immunoglobulins (IgG and IgM) and platelet (PLT) count. APRI and FIB-4 scores were calculated following these formulas $(29,30)$ : APRI $=($ AST/upper limit of normal $) / P L T$ count $\times 100$; FIB-4 $=($ age $\times$ AST $) /\left(\right.$ PLT count $\left.\times \mathrm{ALT}^{1 / 2}\right)$. ALT and AST are both expressed in international units per liter (IU/L) and PLT count is expressed in $10^{\circ}$ cells per liter. The upper limit of normal for AST was 40 IU/L.

\section{Statistical analysis}

All data are expressed as mean \pm standard deviation or median (IQR) as appropriate for continuous variables according to the Kolmogorov-Smirnov test results. Spearman's rank coefficient was applied to measure the association between LSMs and fibrosis stages. A MannWhitney $\mathrm{U}$ test or independent-samples $t$-test was performed to compare the quantitative variables between two categories when appropriate. The areas under the receiver operating characteristic (ROC) curves (AUCs) with $95 \%$ confidence intervals (CIs) were used to evaluating the diagnostic accuracy of the noninvasive predictors in fibrosis staging. AUCs were compared using the method described by Delong et al. (31). Based on the optimal cutoff values that maximized the Youden index, the accuracy, sensitivity, specificity, positive predictive value (PPV), and negative predictive value (NPV) of LSMs in identifying each fibrosis stage were calculated. Logistic regression analysis was carried out to assess the confounding factors affecting LSMs in predicting significant fibrosis $(\geq$ stage 2 ) and cirrhosis (stage 4). Odds ratios (ORs) were also estimated and are presented with $95 \%$ CIs. $\mathrm{P}$ values $<0.05$ were considered statistically significant. All statistical analyses were performed by SPSS software (version 17.0, SPSS, Chicago, IL, USA) or MedCalc (version 10.4, MedCalc Software, Mariakerke, Belgium).

\section{Results}

\section{Patients}

Among the enrolled 121 patients with AILD, 15 of them were not included according to the exclusion criteria, including 2 patients with ages under 18 years old, 4 patients with coexisting other liver diseases, and 9 patients whose serum biomarkers were not completely available within 4 weeks before the biopsy. Hence, 106 AILD patients eligible for further study in STE measurements were included. In the remaining patients, technical failure occurred in 2 patients due to the inability to hold breath, and unreliable results were observed in 2 patients with IQR/ $M$ exceeding $30 \%$. Ultimately, the final analyzed population was 102 subjects with their demographical characteristics, serum biomarkers, and histological results presented in Table 1. The enrolled AILD subjects comprised PBC, AIH, and AIH-PBC overlap syndrome which counted about $47.0 \%$ (48/102), 31.4\% (32/102), and 21.6\% (22/102), respectively. The numbers of patients with AILD at different fibrosis stages were shown in Table 2 .

\section{Liver stiffness measured by STE at each fibrosis stage}

The distribution of STE measured liver stiffness at each fibrosis stage was illustrated in Figure 2 and Table 3. The median LSMs increased with the advancing fibrosis stage. The Spearman's correlation coefficient for LSMs and fibrosis stage was $0.66(\mathrm{P}<0.001)$. The comparison of LSMs between successive fibrosis stages demonstrated that significantly higher LSMs were observed in progressed stages $(\mathrm{P}<0.05$ for all). The AUCs of LSMs in stage $0-1$ versus stage 2 , stage 2 versus stage 3 , stage 3 versus stage 4 were $0.74,0.71$, and 0.78 , respectively (Table 4 ).

\section{Diagnostic capability of LSMs in liver fibrosis staging}

As presented in Figure 3, the AUCs of LSMs in identifying significant fibrosis ( $\geq$ stage 2 ), severe fibrosis ( $\geq$ stage 3 ), and cirrhosis (stage 4) were significantly higher than that of APRI or FIB-4 ( $\geq$ stage 2: 0.82 vs. 0.67 vs. $0.70 ; \geq$ stage 3: $0.87 v s$. 0.64 vs. 0.68 ; stage 4: 0.91 vs. 0.72 vs. $0.75, \mathrm{P}<0.05$ for all). The corresponding cutoff values for staging liver fibrosis were $9.07 \mathrm{kPa}(1.74 \mathrm{~m} / \mathrm{s})$ for stage 2 or higher, $9.97 \mathrm{kPa}$ $(1.82 \mathrm{~m} / \mathrm{s})$ for stage 3 or higher, and $10.48 \mathrm{kPa}(1.87 \mathrm{~m} / \mathrm{s})$ for stage 4 , with accuracy of $80.4 \%, 77.5 \%$, and $76.6 \%$, respectively (Table 5). 
Table 1 Characteristics of the study patients with AILD

\begin{tabular}{|c|c|}
\hline Characteristics & Value $(n=102)$ \\
\hline Age $(y)^{\dagger}$ & $51 \pm 13(19-73)$ \\
\hline Gender, female, $\mathrm{n}(\%)^{\ddagger}$ & 85 [83] \\
\hline BMI $\left(\mathrm{kg} / \mathrm{m}^{2}\right)^{\dagger}$ & $22.4 \pm 2.7(17.7-29.5)$ \\
\hline \multicolumn{2}{|l|}{ Serum biomarkers } \\
\hline ALT (IU/L) & 34.5 (22.0-81.0; 8.0-435.0) \\
\hline AST (IU/L) & 35.5 (27.0-86.0; 10.0-414.0) \\
\hline GGT (IU/L) & $177.5(104.0-335.0 ; 14.0-1,882.0)$ \\
\hline ALP (IU/L) & 119.5 (81.0-225.0; 42.0-3,114.0) \\
\hline TBIL $(\mu \mathrm{mol} / \mathrm{L})$ & $15.6(11.9-25.8 ; 5.9-548.3)$ \\
\hline ALB (g/L) & $44.1(40.7-47.0 ; 25.2-80.2)$ \\
\hline PLT count $\left(10^{9} / \mathrm{L}\right)$ & $131.0(73.0-205.0 ; 29.0-351.0)$ \\
\hline IgG level (g/L) & $18.3(15.4-23.7 ; 8.5-50.2)$ \\
\hline IgM level (g/L) & $\begin{array}{c}2,405.0(1,380.0-3,870.0 \\
160.0-12,900.0)\end{array}$ \\
\hline APRI & $0.97(0.40-1.69 ; 0.16-14.79)$ \\
\hline FIB-4 & 3.08 (1.49-5.83; 0.20-25.78) \\
\hline \multicolumn{2}{|l|}{ Fibrosis stage, $\mathrm{n}[\%]^{\ddagger}$} \\
\hline so & 2 [2] \\
\hline S1 & $33[32]$ \\
\hline $\mathrm{S} 2$ & $37[36]$ \\
\hline S3 & $13[13]$ \\
\hline S4 & $17[17]$ \\
\hline \multicolumn{2}{|l|}{ Activity grade, $\mathrm{n}[\%]^{\ddagger}$} \\
\hline G0 & $0[0]$ \\
\hline G1 & 23 [23] \\
\hline G2 & $44[43]$ \\
\hline G3 & $33[32]$ \\
\hline G4 & $2[2]$ \\
\hline
\end{tabular}

${ }^{\dagger}$, Data are as mean \pm standard deviation, with ranges in parentheses. ${ }^{\ddagger}$, Data are numbers of patients, with counted percentage in parentheses. Unless otherwise indicated, data are median, with IQR and range in parentheses. Fibrosis stage and activity grade were scored according to the Scheuer classification system (28). AILD, autoimmune liver diseases; BMI, body mass index; ALT, alanine aminotransferase; AST, aspartate aminotransferase; GGT, gamma-glutamyltransferase; ALP, alkaline phosphatase; TBIL, total bilirubin; ALB, albumin; PLT, platelet; APRI, aspartate aminotransferase-to-platelet ratio index; FIB-4, fibrosis index based on the four-factor; IQR, interquartile range.

\section{Concordance of STE measured liver stiffness versus Scheuer fibrosis stages}

The concordance of LSMs obtained by STE versus Scheuer stages at each fibrosis stage was shown in Table 6. When using the optimal cutoff values, patients were correctly identified at stage 2 with the highest concordance rate of $81.2 \%$. Lower rates were observed for correctly classifying stage 3 and stage 4, with concordance rates below $50 \%$. Overall, 57 of 102 patients (55.9\%) were correctly classified by STE.

\section{Factors affecting LSMs obtained by STE in the prediction of significant fibrosis or cirrbosis predicting}

According to the logistic regression analysis results, the histological fibrosis stage was the only independent factor associated with STE in the diagnosis of significant fibrosis with an OR of 5.9 (95\% CI: $2.8-12.3, \mathrm{P}<0.001)$. In the diagnosis of cirrhosis by STE, both histological fibrosis stage and serum ALB levels were independently associated variables, with an OR of 4.2 (95\% CI: 2.2-7.8, $\mathrm{P}<0.001$ ), and 0.9 (95\% CI: $0.8-1.0, \mathrm{P}=0.04)$, respectively.

\section{Discussion}

APRI and FIB-4, the well-recognized serologic biomarker scores, demonstrated good accuracy in identifying significant and severe fibrosis in patients with $\mathrm{CHC}(8,9)$. In recent years, these two indexes were also used in assessing other CLD including AILD (10-13,20). In our patients with AILD, the fair diagnostic accuracy of APRI and FIB-4 were observed in identifying cirrhosis (AUCs, 0.72 , and 0.75 , respectively), while the diagnostic accuracy of both APRI and FIB-4 in classifying significant fibrosis (AUCs, 0.67 and 0.70 , respectively) and severe fibrosis (AUCs, 0.64 and 0.68 , respectively) was poor. The results were consistent with previous studies that AUCs of APRI and FIB-4 in predicting significant and severe fibrosis in AILD patients varied from $0.65-0.71(11,20)$. Compared with relatively steady fibrosis stages, the transaminases-related serologic scores were more easily changed by hepatocellular damage, especially in AIH patients. Active hepatitis may overestimate the fibrosis stage. Therefore, the diagnostic performance of APRI and FIB-4 in fibrosis staging for patients with AILD was limited.

Unlike the serum biomarkers, tissue stiffness reflects the biological and mechanical properties that depend mainly 
Table 2 Numbers of the study patients with AILD at different fibrosis stages

\begin{tabular}{lccccc}
\hline Fibrosis stage & S0-1 & S2 & S3 & S4 & Total \\
\hline PBC & 17 & 16 & 7 & 48 & 32 \\
AlH & 7 & 15 & 6 & 4 & 5 \\
AlH-PBC & 11 & 6 & 0 & 13 & 102 \\
Total & 35 & 37 & 13 & 17 \\
\hline
\end{tabular}

AILD, autoimmune liver diseases; PBC, primary biliary cholangitis; AlH, autoimmune hepatitis; AlH-PBC, autoimmune hepatitis-primary biliary cholangitis overlap syndrome.

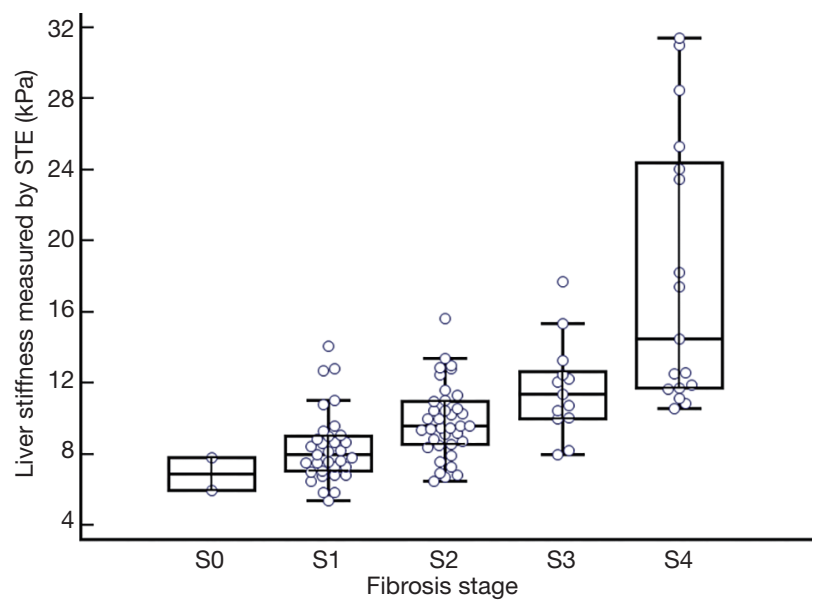

Figure 2 Distribution of liver stiffness values obtained by STE in AILD patients at different fibrosis stages. STE, sound touch elastography; AILD, autoimmune liver diseases.

on its molecular composition and microstructure (32). The stiffness value quantified by SWE is determined by the amount of collagen deposition and microstructure of fiber components in the liver, which has the potential to monitor histopathologic changes. In this study, LSMs substantially increased with the progressed fibrosis stages with a correlation coefficient of $0.66(\mathrm{P}<0.001)$. Also, STE exhibited good diagnostic capability in identifying significant fibrosis, severe fibrosis, and cirrhosis with AUCs of $0.82,0.87$, and 0.91 , respectively. In the classification of fibrosis stages for patients with AILD, similar findings were also reported in another study by using Supersonic shear imaging (SSI), with a correlation coefficient of 0.68 and AUCs of approximately 0.85 (17). Although STE showed good diagnostic performance in discrimination of binary fibrosis stages (e.g., stage 0-2 vs. stage 3-4), its diagnostic accuracy in defining neighboring fibrosis stages (e.g., stage 2 vs. stage 3) seemed inferior, with AUCs of 0.71-0.78.
This is in agreement with the reported limitations of USE in differentiating between individual fibrosis stages $(13,17,24,33)$.

Various cutoff values of staging fibrosis were found in patients with different etiologies by using different elastography platforms. In the current study, the cutoff values for predicting significant fibrosis and cirrhosis were 9.07 and $10.48 \mathrm{kPa}$, respectively, which were higher than that of 7.31 and $9.55 \mathrm{kPa}$ we reported in our recent study in patients with chronic hepatitis B (CHB) (34). Zeng et al. (17) also reported higher cutoff values in patients with AILD than those with CHB or CHC. Thus, simply using cutoff values for CHB in AILD patients, liver fibrosis degree may be upgraded. In the diagnosis of significant fibrosis for AILD patients, another study performed on elastography point quantification (ElastPQ) by Park et al. (20) reported a much lower cutoff point of $5.70 \mathrm{kPa}$ in classifying fibrosis $\geq$ stage 2 . As the interchangeability of different elastography platforms remains controversial $(24,25,33,35-37)$, it is difficult to evaluate the discrepancy of reference values from different devices. Moreover, different from our study, Park et al. enrolled AILD patients without including AIH-PBC overlap syndrome, which has been reported to have slightly higher cutoff values than patients with AIH alone (20).

Referring to the optimal cutoff value of $9.07 \mathrm{kPa}$ in the identification of significant fibrosis, the good diagnostic performance was observed with an accuracy of $80.4 \%$. Thus, as a screening approach, STE might have good capability in discrimination of patients with significant fibrosis, and offer timely recommendations for appropriate treatment. With the corresponding cutoff values in classifying severe fibrosis or cirrhosis, we found high NPVs both above $95 \%$, which indicated excellent capabilities of STE in excluding patients with severe fibrosis or cirrhosis. Nonetheless, the accompanying PPVs were low, especially for the identification of cirrhosis, which was consistent with 
Table 3 Liver stiffness values obtained by STE in patients with AILD

\begin{tabular}{|c|c|c|c|c|c|}
\hline Fibrosis stage & So $(n=2)$ & $\mathrm{S} 1(\mathrm{n}=33)$ & $\mathrm{S} 2(\mathrm{n}=37)$ & S3 $(n=13)$ & $\mathrm{S} 4(\mathrm{n}=17)$ \\
\hline IQR & $5.95-7.83$ & $7.05-9.03$ & 8.56-10.98 & $10.02-12.67$ & $11.70-24.37$ \\
\hline Range & $5.95-7.83$ & $5.37-14.10$ & $6.50-15.62$ & 7.98-17.70 & $10.56-31.40$ \\
\hline SWS (m/s) & 1.51 & 1.63 & 1.79 & 1.95 & 2.20 \\
\hline Range & $1.41-1.62$ & $1.34-2.17$ & $1.47-2.28$ & $1.63-2.43$ & $1.88-3.24$ \\
\hline
\end{tabular}

Results are expressed as median values. STE, sound touch elastography; AILD, autoimmune liver diseases; IQR, interquartile range; SWS, shear wave speed.

Table 4 Comparison of LSMs acquired by STE between successive fibrosis stages

\begin{tabular}{lccr}
\hline Distinction & $\mathrm{S} 0-1(\mathrm{n}=35)$ vs. S2 $(\mathrm{n}=37)$ & $\mathrm{S} 2(\mathrm{n}=37)$ vs. S3 $(\mathrm{n}=13)$ & $\mathrm{S3}(\mathrm{n}=13)$ vs. S4 $(\mathrm{n}=17)$ \\
\hline Fold change & 1.23 & 1.18 & 1.28 \\
P value & $<0.001$ & 0.020 & 0.001 \\
AUC (95\% Cl) & $0.74(0.62-0.83)$ & $0.71(0.56-0.83)$ & $0.78(0.59-0.91)$ \\
\hline
\end{tabular}

Fold change was calculated as the median ratio in the compared successive fibrosis stage cohort. LSM, liver stiffness measurement; STE, sound touch elastography; AUC, area under the receiver operating characteristic curve; $\mathrm{Cl}$, confidence interval.

previous studies $(11,17)$. Finally, 55.9\% of patients with AILD were correctly classified into the exact fibrosis stage, and similar results were reported in the study of Zeng et al. with a concordance rate of $53.5 \%$ in liver fibrosis staging by SSI (17).

In our study cohort, close cutoff values for identifying fibrosis stage 3 or greater, and stage 4 were observed by using STE. This might be related to the comparatively small patient populations in each group. Previous studies of shear wave-based USE in liver fibrosis staging for patients with AILD suggested that the cutoff values for diagnosing cirrhosis varied from 9.28 to $19.00 \mathrm{kPa}(11,17,20,38)$. Except for the different patient populations and the various compositions of fibrosis stages, the large variation of LSMs in cirrhosis patients might also contribute to this diversity across different studies. Furthermore, stiffness values obtained by different elastography techniques are difficult to unify, because the reported parameters, shear wave frequency, and other technical factors are not standardized (39). Whether the cutoff value we set for confirming severe fibrosis or cirrhosis in AILD patients is adequate needs further exploration and verification.

By analyzing factors affecting STE in identifying cirrhosis, we found that fibrosis stages and serum ALB were independent factors associated with STE obtained stiffness. This result is consistent with the study of SSI in fibrosis staging for patients with AILD (17). Apart from ALB, some other biomarkers such as ALT, GGT, and TBIL had also been reported as confounders affecting LSMs in liver fibrosis staging for patients with chronic viral hepatitis $(18,37,40)$. While, the results also varied among different studies. The discrepancies might be explained by the various proportion of patients with markedly elevated serological biomarkers. In our series, patients with strikingly elevated aminotransferase only accounted for a very small proportion, which might limit the study on the influence of serum biomarkers on LSMs in fibrosis staging.

Several limitations of our study should be mentioned. First, no pathological diagnosed primary sclerosing cholangitis (PSC) and IgG4 associated sclerosing cholangitis (IgG4-SC) cases were included. This might result from their low incidences and the preferred diagnostic approach of PSC, which relies mainly on endoscopic retrograde cholangiography instead of clinical biopsy $(41,42)$. Meanwhile, for the small population of patients with AIH, $\mathrm{PBC}$, and overlap syndrome in each subcategory of AILD, we did not analyze separately. Moreover, patients we enrolled were not evenly distributed among the different 

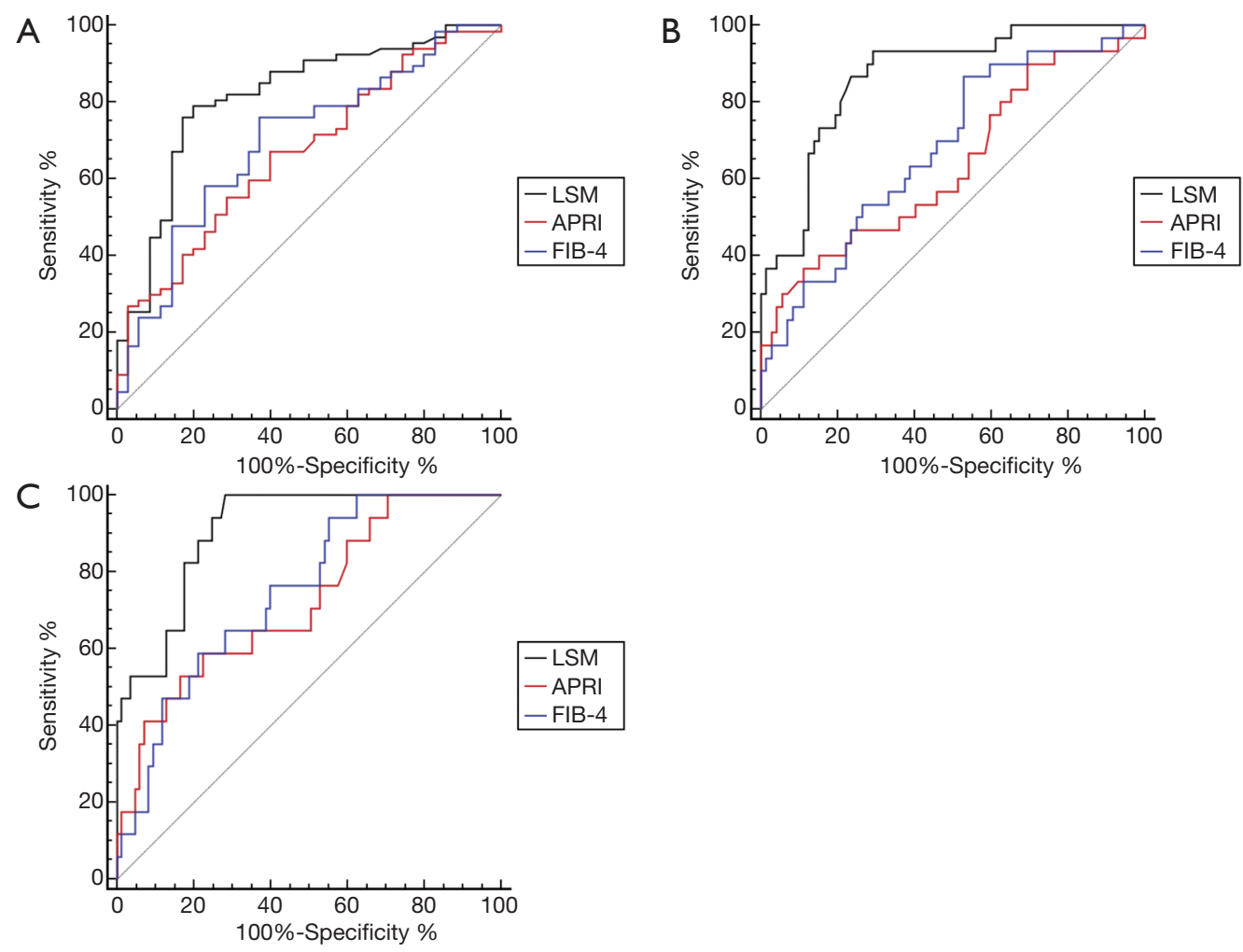

Figure 3 ROC curves of LSMs, APRI, and the four-factor FIB-4 index in the identification of liver fibrosis stages. (A) Comparison of ROC curves for identifying stage 0-1 vs. stage 2-4, P values for LSMs versus APRI or FIB-4 were 0.013 and 0.035 , respectively; (B) comparison of ROC curves for identifying stage 0-2 vs. stage 3-4, P values for LSMs versus APRI or FIB-4 were $<0.001$ and 0.004 , respectively; (C) comparison of ROC curves for identifying stage 0-3 vs. stage 4, P values for LSMs versus APRI or FIB-4 were 0.02 and 0.04 , respectively. ROC, receiver operating characteristic; LSM, liver stiffness measurement; APRI, aspartate aminotransferase-to-platelet ratio index; FIB-4, fibrosis-4.

Table 5 Diagnostic capability of STE in identifying significant fibrosis, severe fibrosis and cirrhosis

\begin{tabular}{|c|c|c|c|c|c|c|c|c|}
\hline Distinction & $P$ value & $\begin{array}{l}\text { AUC }(95 \% \\
\mathrm{Cl})\end{array}$ & $\begin{array}{l}\text { Cutoff } \\
\text { values }\end{array}$ & $\begin{array}{c}\text { Accuracy } \\
(\%)\end{array}$ & $\begin{array}{c}\text { Sensitivity } \\
(\%)\end{array}$ & $\begin{array}{l}\text { Specificity } \\
(\%)\end{array}$ & PPV (\%) & NPV (\%) \\
\hline $\begin{array}{l}\geq \text { Stage } 2 \text { ( } n=67 \text { vs. } \\
\mathrm{n}=35)\end{array}$ & $<0.001$ & $\begin{array}{c}0.82 \\
(0.73-0.89)\end{array}$ & $\begin{array}{c}9.07 \mathrm{kPa} \\
(1.74 \mathrm{~m} / \mathrm{s})\end{array}$ & 80.4 & 79.1 & 80.0 & 88.3 & 66.7 \\
\hline $\begin{array}{l}\geq \text { Stage } 3 \text { ( } n=30 \text { vs. } \\
n=72 \text { ) }\end{array}$ & $<0.001$ & $\begin{array}{c}0.87 \\
(0.78-0.93)\end{array}$ & $\begin{array}{c}9.97 \mathrm{kPa} \\
(1.82 \mathrm{~m} / \mathrm{s})\end{array}$ & 77.5 & 93.3 & 70.8 & 57.1 & 96.2 \\
\hline
\end{tabular}

STE, sound touch elastography; AUC, area under the receiver operating characteristic curve; Cl, confidence interval; PPV, positive predictive value; NPV, negative predictive value.

fibrosis stages. Fewer patients at stage 3 and stage 4 were included. Additionally, not all liver biopsies met the American Society for the Study of Liver Diseases criteria of a sample of at least $2-3 \mathrm{~cm}$ in length with at least 11 complete portal tracts (43); and the interobserver agreement between the two pathologists was not assessed in this study. As only three stiffness values were collected for each patient, this might limit the role of IQR/M in identifying 
Table 6 Concordance rate of liver stiffness measured by STE versus Scheuer fibrosis stages

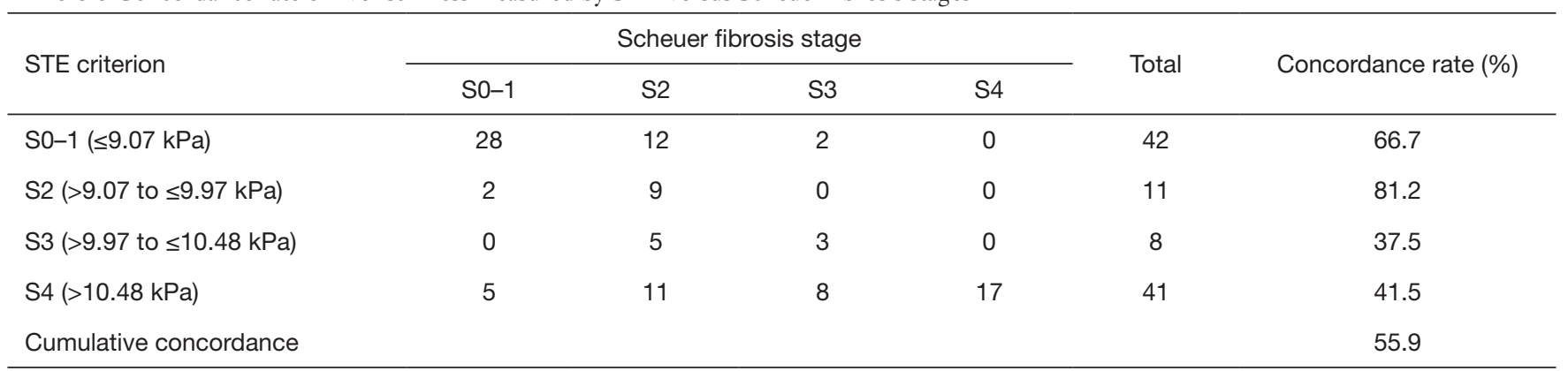

STE, sound touch elastography.

patients with more variable measurements. Finally, no extra series was set as a validation cohort. As the first reported criteria of STE in assessing fibrosis stages in AILD patients, additional validating studies in a large population should be conducted.

\section{Conclusions}

In summary, STE exhibited good diagnostic capability for the evaluation of liver fibrosis stages in patients with AILD. As a noninvasive modality for liver fibrosis staging, STE is superior to fibrosis-related serological APRI and FIB4 indexes. However, the cutoff values of STE measured stiffness in identifying liver fibrosis stages need further validation.

\section{Acknowledgments}

We wish to thank Yue Yu, PhD (Department of Health Science Research, Mayo Clinic) for statistical assistance, Jennifer L. Poston (Department of Biomedical Engineering, Mayo Clinic) and Ao Shi (Center for Regenerative Medicine, Mayo Clinic) for language editorial assistance in the preparation of the manuscript.

Funding: This research was supported by the National Natural Science Foundation of China (Grant No. 81701702) and Sichuan Science and Technology Program (Grant No. 2020YFS0211).

\section{Footnote}

Conflicts of Interest: All authors have completed the ICMJE uniform disclosure form (available at http://dx.doi. org/10.21037/qims-20-521). The authors have no conflicts of interest to declare.
Ethical Statement: This study was approved by the Ethics Committee of West China Hospital with written informed consent from all the patients.

Open Access Statement: This is an Open Access article distributed in accordance with the Creative Commons Attribution-NonCommercial-NoDerivs 4.0 International License (CC BY-NC-ND 4.0), which permits the noncommercial replication and distribution of the article with the strict proviso that no changes or edits are made and the original work is properly cited (including links to both the formal publication through the relevant DOI and the license). See: https://creativecommons.org/licenses/by-nc-nd/4.0/.

\section{References}

1. Tanaka A, Ma X, Yokosuka O, Weltman M, You H, Amarapurkar DN, Kim YJ, Abbas Z, Payawal DA, Chang ML, Efe C, Ozaslan E, Abe M, Mitchell-Thain R, Zeniya M, Han KH, Vierling JM, Takikawa H. Autoimmune liver diseases in the Asia-Pacific region: proceedings of APASL symposium on AIH and PBC 2016. Hepatol Int 2016;10:909-15.

2. Jeong SH. Current epidemiology and clinical characteristics of autoimmune liver diseases in South Korea. Clin Mol Hepatol 2018;24:10-9.

3. Lindor KD, Bowlus CL, Boyer J, Levy C, Mayo M. Primary biliary cholangitis: 2018 practice guidance from the American Association for the study of liver diseases. Hepatology 2019;69:394-419.

4. Mack CL, Adams D, Assis DN, Kerkar N, Manns MP, Mayo MJ, Vierling JM, Alsawas M, Murad MH, Czaja AJ. Diagnosis and management of autoimmune hepatitis in adults and children: 2019 practice guidance and guidelines from the American Association for the study of liver 
diseases. Hepatology 2020;72:671-722.

5. Bravo AA, Sheth SG, Chopra S. Liver biopsy. N Engl J Med 2001;344:495-500.

6. Regev A, Berho M, Jeffers LJ, Milikowski C, Molina EG, Pyrsopoulos NT, Feng ZZ, Reddy KR, Schiff ER. Sampling error and intraobserver variation in liver biopsy in patients with chronic HCV infection. Am J Gastroenterol 2002;97:2614-8.

7. Bedossa $\mathrm{P}$, Dargere D, Paradis V. Sampling variability of liver fibrosis in chronic hepatitis C. Hepatology 2003;38:1449-57.

8. Shaheen AA, Myers RP. Diagnostic accuracy of the aspartate aminotransferase-to-platelet ratio index for the prediction of hepatitis C-related fibrosis: a systematic review. Hepatology 2007;46:912-21.

9. Vallet-Pichard A, Mallet V, Nalpas B, Verkarre V, Nalpas A, Dhalluin-Venier V, Fontaine H, Pol S. FIB-4: an inexpensive and accurate marker of fibrosis in $\mathrm{HCV}$ infection. comparison with liver biopsy and fibrotest. Hepatology 2007;46:32-6.

10. Wang XP, Wang Y, Ma H, Wang H, Yang DW, Zhao $\mathrm{XY}$, Jin EH, Yang ZH. Assessment of liver fibrosis with liver and spleen magnetic resonance elastography, serum markers in chronic liver disease. Quant Imaging Med Surg 2020;10:1208-22.

11. Wu HM, Sheng L, Wang Q, Bao H, Miao Q, Xiao X, Guo CJ, Li H, Ma X, Qiu DK, Hua J. Performance of transient elastography in assessing liver fibrosis in patients with autoimmune hepatitis-primary biliary cholangitis overlap syndrome. World J Gastroenterol 2018;24:737-43.

12. Ucar F, Sezer S, Ginis Z, Ozturk G, Albayrak A, Basar O, Ekiz F, Coban S, Yuksel O, Armutcu F, Akbal E. APRI, the FIB-4 score, and Forn's index have noninvasive diagnostic value for liver fibrosis in patients with chronic hepatitis $\mathrm{B}$. Eur J Gastroenterol Hepatol 2013;25:1076-81.

13. Lu Q, Lu C, Li J, Ling W, Qi X, He D, Liu J, Wen T, Wu H, Zhu $\mathrm{H}$, Luo Y. Stiffness value and serum biomarkers in liver fibrosis staging: study in large surgical specimens in patients with chronic hepatitis B. Radiology 2016;280:290-9.

14. Ma JJ, Ding H, Mao F, Sun HC, Xu C, Wang WP. Assessment of liver fibrosis with elastography point quantification technique in chronic hepatitis B virus patients: a comparison with liver pathological results. J Gastroenterol Hepatol 2014;29:814-9.

15. Ogawa E, Furusyo N, Toyoda K, Takeoka H, Otaguro S, Hamada M, Murata M, Sawayama Y, Hayashi J. Transient elastography for patients with chronic hepatitis B and C virus infection: non-invasive, quantitative assessment of liver fibrosis. Hepatol Res 2007;37:1002-10.

16. Goertz RS, Sturm J, Pfeifer L, Wildner D, Wachter DL, Neurath MF, Strobel D. ARFI cut-off values and significance of standard deviation for liver fibrosis staging in patients with chronic liver disease. Ann Hepatol 2013;12:935-41.

17. Zeng J, Huang ZP, Zheng J, Wu T, Zheng RQ. Noninvasive assessment of liver fibrosis using two-dimensional shear wave elastography in patients with autoimmune liver diseases. World J Gastroenterol 2017;23:4839-46.

18. Zeng J, Liu GJ, Huang ZP Zheng J, Wu T, Zheng RQ, Lu MD. Diagnostic accuracy of two-dimensional shear wave elastography for the non-invasive staging of hepatic fibrosis in chronic hepatitis B: a cohort study with internal validation. Eur Radiol 2014;24:2572-81.

19. Thiele M, Madsen BS, Procopet B Hansen JF, Møller LMS, Detlefsen S, Berzigotti A, Krag A. Reliability criteria for liver stiffness measurements with real-time 2D shear wave elastography in different clinical scenarios of chronic liver disease. Ultraschall Med 2017;38:648-54.

20. Park DW, Lee YJ, Chang W, Park JH, Lee KH, Kim YH, Kang NK, Chung JW, Jang HY, Ahn S, Kim H, Jeong SH, Kim JW, Jang ES. Diagnostic performance of a point shear wave elastography (pSWE) for hepatic fibrosis in patients with autoimmune liver disease. PLoS One 2019;14:e212771.

21. Sporea I, Sirli RL, Deleanu A, Popescu A, Focsa M, Danila M, Tudora A. Acoustic radiation force impulse elastography as compared to transient elastography and liver biopsy in patients with chronic hepatopathies. Ultraschall Med 2011;32:S46-52.

22. Gatos I, Drazinos P, Yarmenitis S, Theotokas I, Zoumpoulis PS. Comparison of sound touch elastography, shear wave elastography and vibration-controlled transient elastography in chronic liver disease assessment using liver biopsy as the "reference standard". Ultrasound Med Biol 2020;46:959-71.

23. Ren X, Xia S, Ni Z, Zhan W, Zhou J. Analysis of three ultrasound elastography techniques for grading liver fibrosis in patients with chronic hepatitis B. Radiol Med 2018;123:735-41.

24. Dietrich CF, Bamber J, Berzigotti A, Bota S, Cantisani V, Castera L, Cosgrove D, Ferraioli G, Friedrich-Rust M, Gilja OH, Goertz RS, Karlas T, de Knegt R, de Ledinghen V, Piscaglia F, Procopet B, Saftoiu A, Sidhu PS, Sporea I, Thiele M. EFSUMB guidelines and recommendations on the clinical use of liver ultrasound elastography, update 2017 (long version). Ultraschall Med 2017;38:e16-47. 
25. Barr RG, Ferraioli G, Palmeri ML, Goodman ZD, GarciaTsao G, Rubin J, Garra B, Myers RP, Wilson SR, Rubens D, Levine D. Elastography assessment of liver fibrosis: society of radiologists in ultrasound consensus conference statement. Radiology 2015;276:845-61.

26. Palmeri ML, Nightingale KR. Acoustic radiation forcebased elasticity imaging methods. Interface Focus 2011;1:553-64.

27. Lin LI. A concordance correlation coefficient to evaluate reproducibility. Biometrics 1989;45:255-68.

28. Desmet VJ, Gerber M, Hoofnagle JH, Manns M, Scheuer PJ. Classification of chronic hepatitis: diagnosis, grading and staging. Hepatology 1994;19:1513-20.

29. Wai CT, Greenson JK, Fontana RJ, Kalbfleisch JD, Marrero JA, Conjeevaram HS, Lok AS. A simple noninvasive index can predict both significant fibrosis and cirrhosis in patients with chronic hepatitis C. Hepatology 2003;38:518-26.

30. Sterling RK, Lissen E, Clumeck N, Sola R, Correa MC, Montaner J, S Sulkowski M, Torriani FJ, Dieterich DT, Thomas DL, Messinger D, Nelson M; APRICOT Clinical Investigators. Development of a simple noninvasive index to predict significant fibrosis in patients with $\mathrm{HIV} / \mathrm{HCV}$ coinfection. Hepatology 2006;43:1317-25.

31. DeLong ER, DeLong DM, Clarke-Pearson DL. Comparing the areas under two or more correlated receiver operating characteristic curves: a nonparametric approach. Biometrics 1988;44:837-45.

32. Hui CK, Leung N, Yuen ST, Zhang HY, Leung KW, Lu L, Cheung SK, Wong WM, Lau GK. Natural history and disease progression in Chinese chronic hepatitis $\mathrm{B}$ patients in immune-tolerant phase. Hepatology 2007;46:395-401.

33. Sigrist RMS, Liau J, Kaffas AE, Chammas MC, Willmann JK. Ultrasound elastography: review of techniques and clinical applications. Theranostics 2017;7:1303-29.

34. Yang L, Li J, Ma L, Xiang H, He D, Lu C, Tang L, Luo Y, Chen S. Noninvasive assessment of liver fibrosis in chronic hepatitis B carriers with sound touch elastography: study of surgical pathology specimens. Expert Rev Med Devices 2020;17:845-53.

35. Sigrist RMS, El Kaffas A, Jeffrey RB, Rosenberg J,

Cite this article as: Yang L, Ling W, He D, Lu C, Ma L, Tang L, Luo Y, Chen S. Shear wave-based sound touch elastography in liver fibrosis assessment for patients with autoimmune liver diseases. Quant Imaging Med Surg 2021;11(4):1532-1542. doi: 10.21037/qims-20-521
Willmann JK. Intra-individual comparison between 2-D shear wave elastography (GE system) and virtual touch tissue quantification (siemens system) in grading liver fibrosis. Ultrasound Med Biol 2017;43:2774-82.

36. Gilligan LA, Trout AT, Bennett P, Dillman JR. Repeatability and agreement of shear wave speed measurements in phantoms and human livers across 6 ultrasound 2-dimensional shear wave elastography systems. Invest Radiol 2020;55:191-9.

37. Cassinotto C, Lapuyade B, Mouries A, Hiriart JB, Vergniol J, Gaye D, Castain C, Le Bail B, Chermak F, Foucher J, Laurent F, Montaudon M, De Ledinghen V. Non-invasive assessment of liver fibrosis with impulse elastography: comparison of Supersonic Shear Imaging with ARFI and FibroScan. J Hepatol 2014;61:550-7.

38. E Anastasiou O, Büchter M, A Baba H, Korth J, Canbay A, Gerken G, Kahraman A. Performance and utility of transient elastography and non-invasive markers of liver fibrosis in patients with autoimmune hepatitis: a single centre experience. Hepat Mon 2016;16:e40737.

39. Tang A, Cloutier G, Szeverenyi NM, Sirlin CB. Ultrasound elastography and MR elastography for assessing liver fibrosis: Part 2, diagnostic performance, confounders, and future directions. AJR Am J Roentgenol 2015;205:33-40.

40. Arena U, Vizzutti F, Corti G, Ambu S, Stasi C, Bresci S, Moscarella S, Boddi V, Petrarca A, Laffi G, Marra F, Pinzani M. Acute viral hepatitis increases liver stiffness values measured by transient elastography. Hepatology 2008;47:380-4.

41. Boonstra K, Beuers U, Ponsioen CY. Epidemiology of primary sclerosing cholangitis and primary biliary cirrhosis: a systematic review. J Hepatol 2012;56:1181-8.

42. Andraus W, Haddad L, Nacif LS, Silva FD, Blasbalg R, D'Albuquerque LA. The best approach for diagnosing primary sclerosing cholangitis. Clinics (Sao Paulo) 2011;66:1987-9.

43. Rockey DC, Caldwell SH, Goodman ZD, Nelson RC, Smith AD; American Association for the Study of Liver Diseases. Liver biopsy. Hepatology 2009;49:1017-44. 